\title{
Lidil
}

Revue de linguistique et de didactique des langues

$63 \mid 2021$

Littératie numérique et didactique des langues et des cultures

\section{Le savoir ponctuer au lycée professionnel : attendus, acquis et perspectives d'enseignement}

Knowing How to Punctuate in Vocational High Schools: Expectations, Achievements and Teaching Perspectives

Cindy De Amaral et Véronique Paolacci

(2) OpenEdition

Journals

Édition électronique

URL : http://journals.openedition.org/lidil/8858

DOI : $10.4000 /$ lidil.8858

ISSN : 1960-6052

Éditeur

UGA Éditions/Université Grenoble Alpes

Édition imprimée

ISBN : 978-2-37747-283-3

ISSN : $1146-6480$

Référence électronique

Cindy De Amaral et Véronique Paolacci, « Le savoir ponctuer au lycée professionnel : attendus, acquis et perspectives d'enseignement », Lidil [En ligne], 63 | 2021, mis en ligne le 30 avril 2021, consulté le 30 avril 2021. URL : http://journals.openedition.org/lidil/8858; DOI : https://doi.org/10.4000/lidil.8858

Ce document a été généré automatiquement le 30 avril 2021.

(C) Lidil 


\title{
Le savoir ponctuer au lycée professionnel : attendus, acquis et perspectives d'enseignement
}

\author{
Knowing How to Punctuate in Vocational High Schools: Expectations, \\ Achievements and Teaching Perspectives
}

Cindy De Amaral et Véronique Paolacci

\begin{abstract}
Remerciements
Nous remercions les relecteurs de la revue pour leurs avis et leurs conseils précieux dans le processus de réécriture de cet article.
\end{abstract}

\section{Introduction}

1 Les enseignants, comme la recherche (Guernier, 2008 ; Guernier et coll., 2017), font état de difficultés accrues rencontrées à l'écrit par les élèves de lycée professionnel (LP) ${ }^{1}$. Écrire est en effet une tâche complexe qui mobilise de nombreuses compétences. Si plusieurs études font état des difficultés rencontrées par le public de LP dans les domaines orthographiques et grammaticaux (Guernier et coll., 2017; Joyet \& Plissonneau, 2017), peu de recherches se sont intéressées à ce jour aux performances des élèves de lycée professionnel ailleurs que dans le domaine strictement orthographique alors même que les observations que nous avons menées lors de notre expérimentation (Jacques \& De Amaral, 2018) nous laissent à penser qu'analyser la manière dont ces élèves ponctuent ou non leurs textes nous donnerait un accès privilégié à leur savoir-faire rédactionnel mais aussi, à leur rapport à l'écrit.

2 Nous nous intéressons donc, dans une perspective de valorisation des compétences ignorées (Penloup, 2007), aux savoir-faire et aux savoirs mis en œuvre par ces élèves. Nous espérons également que la manière dont ces lycéens professionnels ponctuent leurs productions et la manière dont leur enseignante les évalue pourront apparaitre comme un révélateur des effets à moyen terme des pratiques d'enseignement. 
3 Après avoir dressé un premier état des lieux de la didactique de l'écrit au lycée professionnel, de ses enjeux concernant le savoir ponctuer et précisé les choix méthodologiques de notre étude, nous questionnerons les outils didactiques travaillés par l'enseignante observée avant d'analyser les productions écrites collectées en classe. Nous dégagerons à l'issue de cette analyse quelques pistes didactiques fondées sur les compétences des élèves et les pratiques effectives de l'enseignante.

\section{Le savoir ponctuer au lycée professionnel : éléments de problématisation}

4 La sociologie de l'éducation, qui s'est beaucoup intéressée aux élèves de LP pour leur profil sociologique (puisque le LP concentre davantage d'élèves issus de l'immigration et/ou de milieu populaire), laisse à penser qu'il y aurait un lien entre les difficultés scolaires rencontrées par ces élèves et leur rapport à l'écrit. Certains élèves ont davantage de difficultés à adopter le type de rapport à la langue et à l'écrit attendu par l'école (Lahire, 2008). Il leur est notamment plus compliqué d'adopter une certaine distance réflexive vis-à-vis de la langue et de l'objet texte. Charlot (1999) dit de ces lycéens qu'ils entretiennent un rapport plus « oral » aux savoirs que celui attendu par notre système scolaire, ce qui les place en situation d'échec ou de "malentendu " (Bautier \& Rayou, 2014) lorsqu'il s'agit de décrypter les attentes de leurs enseignants dans certaines activités scolaires. Ces éléments induisent la nécessité d'adopter une approche à la fois plus explicite et plus réflexive de l'enseignement dispensé à ce public particulier au travers d'activités métalinguistiques (Maynard et coll., 2018) et métascripturales ${ }^{2}$. Or, les recherches menées jusque-là sur le rapport à l'écrit de ces élèves laissent à penser que ces derniers, tout comme leurs enseignants, se focalisent sur des aspects de surface au détriment de l'objet texte comme unité (De Amaral, à paraitre; Guernier, 2008). Une analyse du savoir ponctuer dont ils font preuve pourrait venir éclairer ou nuancer cette idée. Nous postulons en effet que la ponctuation, par bien des aspects, constitue un point nodal de la compétence scripturale et pourrait donc venir nous offrir un éclairage intéressant quant aux compétences rédactionnelles des élèves de lycée professionnel.

\section{Ponctuation et enseignement de la compétence scripturale}

\subsection{Le rôle de la ponctuation dans la " compétence scripturale » (Dabène, 1991) des élèves}

5 Notre analyse s'inscrit dans une approche complète de la « compétence scripturale » au sens où la définit Dabène (1991). Il s'agit donc pour nous de prendre en compte différentes composantes du savoir écrire des élèves parmi lesquelles figurent des dimensions techniques (des savoir-faire) mais aussi des savoirs notionnels (sur la langue notamment) ainsi que des représentations sur l'écrit comme sur l'activité d'écriture (Lafont-Terranova, 2009), qui participent du rapport que chaque élève entretient avec l'écrit. Les études menées jusqu'alors sur le rapport que les élèves entretiennent à l'écrit (tant sur le plan cognitif qu'affectif) montrent qu'un 
changement dans les représentations ou les attitudes constitutives de ce "rapport à » peut avoir des effets didactiques importants (Barré-De Miniac, 2008). Nous partons donc du postulat que la ponctuation, parce qu'elle met en jeu des savoir-faire et des savoirs importants notamment sur le plan syntaxique qui nous intéresse particulièrement ici compte tenu du profil des élèves de LP, mais aussi, parce qu'elle constitue une voie d'accès privilégiée aux représentations que ces élèves se font de l'objet de texte, est un élément essentiel de leur compétence scripturale.

6 Depuis les travaux de l'équipe de Catach des années 1980, les études et les recherches (linguistiques, stylistiques et didactiques) sur la ponctuation sont nombreuses ${ }^{3}$. La question centrale posée est bien celle que présente Dalhet (2003): "Qu'est-ce qui relève de la langue, de son système et qu'est-ce qui relève du sujetscripteur?» (p.123) En d'autres termes, qu'est-ce qui relève de la norme et de la variation illustrée par les scripteurs? Ces questions sont au cœur de notre étude qui met en parallèle l'attente normée de la professeure, soucieuse de communiquer la structure ritualisée de l'écrit produit, la lettre (Jagueneau, 2000, p. 191), et les usages rédactionnels de ses élèves.

\subsection{Une vue d'ensemble du système de ponctuation : de la virgule au blanc graphique}

$7 \mathrm{Au}$ regard du genre particulier étudié, la lettre de réclamation, nous adoptons une approche assez large du système ponctuationnel. Nous partons de la typologie de Tournier (1980) qui considère la ponctuation de mot, de phrase, de texte et la ponctuation métagraphique. La ponctuation rejoint à ce propos la typographie et la "matérialité graphique » selon la théorie d'Anis (Dalhet, 2003, p. 24) en n'étant pas oralisée. L'empan du paradigme de la ponctuation est donc assez important, mais les usages des élèves que nous observons dans les recherches (Paolacci \& Rossi-Gensane, $2016,2019)$ renvoient à un usage plutôt limité de signes : les signes de ponctuation forte que sont les différents points(?!), la virgule et les signes du discours direct. Jagueneau (2000), à partir d'un corpus épistolaire ordinaire, montre que les points (accompagnés ou non de majuscules), les virgules ainsi que les alinéas font système.

\subsection{Les fonctions syntaxiques et sémantiques de la ponctuation}

8 Vedenina (1980) a modélisé les fonctions syntaxiques et sémantiques de la ponctuation qui participent ainsi à la cohésion textuelle. Ses différents rôles syntaxiques se conjuguent avec une fonction sémantique. Si l'on prend l'exemple de la virgule, qui est le signe le plus tardivement acquis par les scripteurs selon les travaux en psychologie du langage (Fayol, 1997), les fonctions syntaxiques de ce signe sont diverses. On peut s'entendre sur quelques emplois normés que relayent les ouvrages didactiques (Paolacci et coll., 2016, p. 234) : la juxtaposition d'unités syntaxiques (énumération), le détachement d'éléments comme les compléments de phrase, le détachement des phrases incises ou l'effacement d'énoncés. Ces rôles syntaxiques de la virgule sont liés à un rôle sémantique : elle peut, selon sa place dans la phrase, faire varier le sens de l'énoncé4. 


\subsection{La fonction pragmatique de la ponctuation}

Nous nous positionnons par rapport à une fonction pragmatique de la ponctuation qui complète ses fonctions syntaxiques et sémantiques. L'étude de Romain et coll. (2016) montre, dans le même contexte de la lettre de réclamation, comment des rédacteurs professionnels prennent en compte le destinataire et comment les modifications apportées à la lettre de départ visent une plus grande lisibilité des propos (gras, retours à la ligne, etc.). Le blanc du texte dans une approche plus macro de la lettre a une fonction particulière, on parlera alors de ponctuation blanche ${ }^{5}$ (Eluerd, 2017). Les retours à la ligne précédés de blancs respectent un découpage du texte voulu par le scripteur et en facilitent la lecture.

De façon générale, tout scripteur par son écriture révèle une intention de communication. En étant visible, le texte devient lisible et la ponctuation contribue à cette vi-lisibilité (Anis, 1983). Ces postulats s'adaptent bien au genre épistolaire qui vise un effet sur le destinataire. Notre étude, dans l'approche qu'elle propose de la ponctuation, tente donc de tenir compte à la fois des enjeux pragmatiques liés au genre professionnel de la lettre de réclamation et de la situation didactique qui induit une certaine relation au lecteur-évaluateur.

\section{Méthodologie de l'étude}

11 Nos choix méthodologiques visent une description fine des compétences rédactionnelles des élèves de LP à travers une analyse de leurs productions effectives alors que nous sommes conscientes de la difficulté dans le fait même de faire produire des textes à certains de ces élèves, vraiment "fâchés » avec l'écrit (Barré-De Miniac, 2001). Compte tenu de la situation de dissymétrie que la ponctuation induit entre l'oral et l'écrit, il nous semble particulièrement intéressant de nous demander comment ponctuent les élèves observés, supposés entretenir un rapport plus " oral » qu' "écrit » aux savoirs. Nous avons donc souhaité exploiter les textes produits lors de la phase de posttest de notre expérimentation (nous avons collecté trente écrits) pour nous livrer à une analyse fine du savoir ponctuer mis en œuvre par ces élèves, au travers de quelques extraits de leurs productions qui nous semblent illustratifs.

\subsection{Profil des élèves et de l'enseignante observés}

Les trente auteurs de ces textes sont en classe de première gestion-administration, une filière tertiaire de LP qui scolarise majoritairement des filles (âgées de 16 à 18 ans) et une forte proportion d'élèves en « retard scolaire » (Cnesco \& Ciep, 2016).

L'enseignante observée est une enseignante des disciplines professionnelles très expérimentée (plus de vingt ans d'ancienneté). Nous postulons que les évaluations critériées qu'elle livre des productions d'élèves dans le cadre de notre étude sont assez représentatives de sa pratique habituelle.

\subsection{Contexte : le projet Écrire@lp}

14 Le corpus analysé dans cette étude a été produit dans le cadre d'un projet de recherche plus global: le projet Écrire@lp. Ce projet visait, après une étude diagnostique, à 
concevoir et à tester un dispositif adapté d'enseignement de l'écrit. Les textes analysés dans cette étude ont été produits dans la phase de posttest de cette étude expérimentale et sont extraits de la tâche d'écriture proposée par l'enseignante en clôture du test.

\subsection{Le corpus de notre étude : des lettres de réclamation} sur ordinateur et incluaient des exercices de langue (dont un exercice de ponctuation) et des tâches de production écrite. La tâche finale de ces tests était une production numérique d'écrit professionnel conçue et évaluée par l'enseignante.

Pour cette tâche finale, passée au terme de l'année de la classe de première, l'enseignante a choisi de faire produire une lettre de réclamation, genre qui, selon elle, devait être partiellement maitrisé à ce stade de la formation puisque d'autres types de correspondance assez proches avaient été enseignés au cours de l'année. Notre corpus se compose donc de trente lettres de réclamation dont vingt-sept ${ }^{6}$ ont été évaluées et annotées par l'enseignante, c'est donc sur ces vingt-sept productions notées que nous concentrerons prioritairement notre analyse. Ces lettres numériques rédigées par des apprenants, qui constituent notre objet d'étude, sont le reflet d'une situation énonciative particulière : dans le cadre de la situation de classe, elles ne sont lues que par l'enseignante. Dans cet exercice, qui vise des compétences d'ordre professionnel, le contrat d'évaluation entre enseignant et élèves est explicite. Ces derniers pour ce travail de rédaction doivent respecter un jeu de rôle qui consiste à prendre la place d'un client non satisfait (on pourra alors se référer à la consigne d'écriture qui est mise en annexe 3).

\subsection{Des critères pour une analyse linguistique des usages de la ponctuation dans les lettres de réclamation des élèves}

- Concernant la fonction syntaxique de la ponctuation : le respect des normes grammaticales notamment dans l'emploi des signes de ponctuation forte les plus fréquents tels que le point et la virgule ou une éventuelle proximité avec les usages "ordinaires" de ces signes identifiés par Jagueneau (2000) dans un corpus épistolaire ;

- Concernant la fonction pragmatique de la ponctuation : un emploi jugé comme efficace des blancs typographiques et des alinéas dans une visée de communication (Romain et coll., 2016), un découpage du texte en paragraphes qui semble répondre à une logique textuelle qui tienne compte du lecteur;

- Du point de vue du genre textuel visé: une analyse des marques typographiques (notamment des blancs) représentatives du genre épistolaire.

\subsection{Les évaluations faites par l'enseignante}

Parallèlement à cette analyse linguistique des textes d'élèves, nous nous intéressons également à l'évaluation que l'enseignante a pu en livrer (dans le cadre initial du projet) au travers de grilles d'évaluation et d'annotations manuscrites.

données recueillies auprès de l'enseignante sont donc constituées d'une note (sur 20) accompagnée d'une grille d'évaluation et de diverses annotations que nous 
mettrons ponctuellement en parallèle avec notre analyse du texte. La note attribuée par l'enseignante est indiquée dans la légende de chaque extrait commenté.

\section{Que nous disent les outils didactiques de l'enseignante de ses attentes en matière de «ponctuation »?} d'évaluation utilisée par l'enseignante et la tâche prescrite nous donnent un accès privilégié à l'habitus professionnel de la professeure: ce manuel est en effet régulièrement utilisé en classe et conforme au contenu comme à l'esprit du référentiel $\mathrm{du}$ baccalauréat professionnel de la filière depuis l'introduction des «ateliers rédactionnels » en 2012. L'analyse de ces documents enseignants nous permet donc de venir éclairer les annotations manuscrites faites sur les copies, de nous renseigner indirectement sur les pratiques de classe, mais aussi, et surtout, de reconstituer un certain nombre d'exigences spécifiques à la filière gestion-administration à l'aune desquelles nous pouvons analyser les productions des élèves.

\subsection{Des attendus pas toujours explicites concernant la ponctuation}

Les grilles d'évaluation de l'enseignante (cf. annexe 3) ne comportent pas d'items spécifiques dédiés à la ponctuation. Les pages du manuel consacrées au genre de la lettre de réclamation dont est extraite la tâche proposée ici ne font pas non plus explicitement mention de la ponctuation. Pourtant, si l'on observe certains items de la grille en les mettant en parallèle avec la définition du genre d'écrit visé proposée par le manuel, on peut relever un certain nombre de critères d'évaluation implicitement liés au savoir ponctuer des élèves.

\subsection{Le modèle de la lettre de réclamation : des attendus typographiques}

La séquence didactique proposée par le manuel ${ }^{8}$ (Addis et coll., 2016) s'organise autour d'une visée pragmatique claire, voire d'une dimension perlocutoire de l'acte de langage "réclamer». De cette recherche d'efficacité communicative découlent des attentes formelles qui devraient se traduire par des marqueurs typographiques dans les productions des élèves. En effet, le manuel stipule que la lettre de réclamation doit comprendre trois parties censées correspondre à la visée argumentative du texte. Cette exigence structurelle, qui devrait donc se traduire par des alinéas dans les textes d'élèves, semble trouver sa correspondance dans l'item "construction » de la grille de l'enseignante qui précise que le texte doit être « en grande partie structuré (paragraphe) et cohérent» avec un "enchainement assez logique des éléments essentiels ». La mise en page des lettres modèles du manuel laisse à penser que les règles de mise en page associées au genre épistolaire (et les blancs graphiques qu'elles supposent) font également partie intégrante des critères de réussite pour cet exercice. L'item "normes de présentation » constitue en effet la première entrée de la grille d'évaluation de l'enseignante. 


\subsection{Quelle prise en compte du rôle syntaxique des signes de ponctuation?}

22 Le manuel fait aussi état d'un certain nombre d'attentes sur le plan linguistique (en termes de syntaxe notamment) sans jamais évoquer la ponctuation. La grille d'évaluation de l'enseignante stipule également des critères de "correction de la langue » et sanctionne les "erreurs de syntaxe », mais l'emploi erroné de signes de ponctuation n'est pas explicitement évoqué comme entrant dans cet item. Certaines annotations de l'enseignante laissent à penser qu'une partie des signes de ponctuation pourraient être abordés comme participant d'une norme graphique.

23 L'analyse des items de la grille d'évaluation de l'enseignante, mise en parallèle avec les attendus présentés par le manuel dont cette grille est issue, prouve qu'il existe un paradoxe en ce qui concerne le savoir ponctuer en lycée professionnel. En effet, bien que le manuel et l'enseignante ne fassent pas explicitement mention de la ponctuation comme objet d'apprentissage ou d'évaluation en soi, certaines composantes du savoir ponctuer semblent bien entrer en jeu dans l'évaluation de ces lettres de réclamation. Le savoir ponctuer pourrait alors constituer une forme d'impensé didactique alors même qu'il entre implicitement en jeu dans la compétence scripturale des élèves de LP.

Notre étude nous permet d'observer un contraste important des compétences rédactionnelles de ces élèves qui se traduit à différents niveaux. Nous allons envisager tour à tour les fonctions syntaxiques et pragmatiques de la ponctuation. Pour chaque axe d'analyse considéré, nous nous attacherons à mettre en regard nos commentaires avec l'évaluation de la professeure.

\section{Résultats saillants de notre étude : des acquis contrastés chez les élèves}

25 Les emplois des signes de ponctuation par les élèves de l'étude illustrent des compétences rédactionnelles diverses.

\subsection{Une fonction syntaxique de la ponctuation globalement bien acquise...}

26 Les phénomènes de sous-ponctuation sont peu nombreux dans notre corpus, et ce constat est tout à fait cohérent au vu de l'âge des scripteurs. Le signe " point final » est en général bien présent dans la plupart des lettres. Le phénomène de surponctuation qui divise une même phrase syntaxique (tel qu'illustré dans l'exemple suivant) est assez rare dans le corpus :

Suite à la réception de la commande du 11/09/2018. Nous avons remarqué une erreur sur la facture $1235 \mathrm{du}$ au pourcentage de remise. (IIOY, évaluée à 7) ${ }^{9}$

La ponctuation employée est assez diversifiée, ce qui témoigne d'une connaissance du paradigme de la ponctuation dans son ensemble. Nous relevons l'emploi des deux points et du point-virgule dans une même lettre. Or, même si les emplois des signes ci-dessous restent non normés, nous pouvons valoriser cette production, comme si l'élève s'essayait à différents signes de ponctuation : 
Figure 1. - Capture d'écran de copie annotée (III10X, évaluée à 9).

\section{Monsieur LEGRAND}

Bonjours. nous avons bien reçu votre livraison du vendredi 11 mai et avons pu constater une erreur sur la facture $\mathrm{N}^{\circ} 123 \%$ :

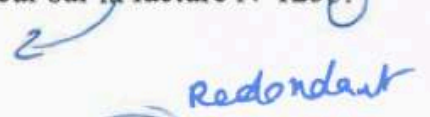

J'ai pu constater que la seeiété Burortro ne nous a pas accordé la remise de $15 \%$ octroyée pour toute commande supérieure à 1000 euros HT.

Merci de bien vouloir rectifier cette erreur sur la facture $\mathrm{N}^{\circ} 1235$;

Cordialement veủillez agréer Madame, Monsieur nos salutations distingués

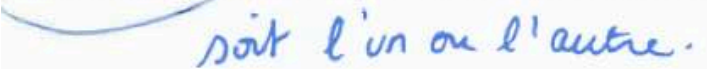

Attachons-nous à présent à la virgule. Les virgules sont aisément utilisées par ces lycéens et cela de façon très variée. Ces signes, qui interagissent avec les points - même dans les écrits d'apprenants plus jeunes - sont à la fois les plus complexes, mais aussi les plus prisés des signes. Bessonnat (1991) les qualifie d'« archiponctèmes». La virgule détache le complément temporel dans l'exemple suivant :

En date du 11 mai 2018, nous avons reçu la facture $N^{\circ}$ 1235. (I0X, évaluée à 9)

La virgule acquiert une fonction de ponctuation forte dans un écrit fortement noté (19 sur 20) par l'enseignante :

Figure 2. - Capture d'écran d'une copie annotée (I9X, évaluée à 19).

Madame, monsieur,

Nous avons reçu notre commande de notre nouveau bureau, cependant nous avons constaté sur la facture que vous n'aviez pas accordé la remise de $15 \%$ sur notre commande qui est supérieure à 1000 euros.

Je vous sereż gyé de bien vouloir nous envoyer une seconde facture et de bien vouloir nous accorder la temise de $15 \%$.

\section{2. ... malgré quelques difficultés observables}

En revanche, des difficultés d'emplois de la ponctuation, qui ne sont pas toujours sanctionnées par l'enseignante, apparaissent dans certains écrits. Nous notons des absences de virgules comme dans l'exemple ci-dessous : 
Figure 3. - Capture d'écran d'une copie annotée (15X, évaluée à 13).

J'ai bien reçu à ce jour, le vendredi 11 mai le bureau que nous vous avions commanđés.

Jai pu constaté une erreur sur la facture $\mathrm{N}^{\circ} 1235$. En effet il était dit que pour toute commande supérieure à $1000 \mathrm{H}$.T nous avions une remise accordée de $15 \%$. Notre commande n'a pas eu le droit à cette remise.

Selon la norme dont nous avons rappelé quelques éléments plus haut, on aurait pu attendre une virgule après « le vendredi 11 mai », apposé à " ce jour ", mais aussi après le connecteur «en effet" et pour isoler le complément "pour toute commande supérieure à $1000 \mathrm{HT}$ ». Ceci n'est pas relevé par l'enseignante qui surligne avec un feston «il était dit». De plus, comme nous l'avions noté dans nos travaux antérieurs (en particulier dans des écrits d'étudiants de master, Paolacci \& Rossi-Gensane, 2019), nous relevons le phénomène de la présence de la virgule entre le sujet et son verbe :

En effet la remise de $15 \%$ pour toute commande supérieure à 1000 euros HT, n'a pas été accordée. (I11X, évaluée à 12)

On pourrait alors se questionner sur le poids de la tradition scolaire qui semble illustrée ici : la fonction prosodique de la ponctuation est mise en avant comme s'il existait une biunivocité entre les signes de ponctuation et les unités de souffle de la voix (dans le cas de l'oralisation d'un texte). Dans le cas qui précède, on pourrait accepter que le sujet long entrainant une pause orale avant le verbe ${ }^{10}$ soit suivi par une virgule.

En outre, certains écrits peu valorisés par l'enseignante présentent l'absence systématique des majuscules (qu'entoure l'enseignante) comme dans l'extrait de la lettre suivante :

Figure 4. - Capture d'écran d'une copie annotée (112X, évaluée à 8).

bonjour Monsieur Legrand,

on vous informe que on nous a pas accorder ya remise de $15 \%$ sur el facture $n^{\circ} 123$. on

souhaiterait que yous nous envoyer une kervelle facture avec la remise de $15 \%$.

cordialement

4 Il nous faudrait interroger l'enseignante sur ses représentations de la ponctuation et de la norme scripturale si l'on souhaite mieux comprendre la valeur qu'elle accorde à l'absence de majuscules dans cet écrit, mais nous postulons que l'usage des majuscules et des points finaux pourrait participer selon elle d'une norme orthographique (au même titre que les accords...).

La ponctuation minimale de la lettre qui précède combinée avec une orthographe défaillante mise en exergue par l'enseignante révèle ce que nous notions plus haut : la ponctuation et ses sous-emplois seraient donc significatifs d'un rapport à l'écrit problématique au niveau orthographique et typographique? Ce serait un constat à vérifier dans une étude plus quantitative.

De plus, le point d'interrogation est fréquemment oublié par les scripteurs observés. 
Figure 5. - Capture d'écran d'une copie annotée (III8X, évaluée à 8).

Nous avions reçus le bureau que nous avions commander chez vous, le vendredi 11 mai, nous avons constatais une anomalie sur la facture $\mathrm{N}^{\circ} 1235$, vous n'aviez pas accordé la remise de $15 \%$ pour toutes les commandes supérieure à 1000 euros. Nous aimerions obtenir une

\subsection{Une fonction pragmatique de la ponctuation plus en demi-teinte?}

37 Penchons-nous sur les blancs graphiques des écrits observés qui apparaissent sous la forme de sauts de lignes et donc de mises en paragraphes isolés par les blancs. Même si certaines lettres sont en un seul bloc (voir texte en annexe 3), les sauts de lignes sont une constante dans la plupart de ces écrits. Ainsi, un découpage très efficace du texte va de pair avec un contenu répondant aux attentes et est donc très positivement évalué par l'enseignante :

Figure 6. - Capture d'écran d'une copie annotée (I12X, évaluée à 18).

Madame,Monsieur

Je fais suite à votre facture qui nous a été adressée en date du 11 Mai.

Nous vous envoyons cette lettre pour vous frevenir que vous nous avons pas eux de remise de $15 \%$ octroyée pour toute commande supérieure à 1000 euros HT sur notre facture $n^{\circ} 1235 \mathrm{eh}$ date du 10 Mai $201 \mathrm{~N}$.

$$
\text { ons }
$$

Nous vous demandons de vous rappeler que vous êtes dans l'obligation légale de respecter les informations indiquées sur notre contrat. Aussi je vous saurai gré de bien vouloir me remettre. une nouvelle facture et une ristourne.

\section{avec}

Dans l'attente de votre réponse, je vous prie de croire, Madame, Monsieur, mes salutations distinguées.

Nous relevons également de nombreuses virgules dans les formules de politesse du début, mais aussi de fin de lettre comme le montre l'exemple ci-après (et cela dans la plupart des lettres): Veuillez accepter, Monsieur, nos salutations les plus sincères (IOX, évaluée à 18). Ces usages, intégrés dans les formules de politesse et souvent rappelés dans le système scolaire, participent selon nous à des fonctions pragmatiques (couplées à des fonctions syntaxiques) de la ponctuation.

Toutefois, nous pouvons nous demander si le découpage en paragraphes est vraiment " pensé » par les élèves; autrement dit, nous ne savons pas si le retour à la ligne n'est pas le résultat d'un automatisme de l'ordre de la typographie: «Je finis une phrase, j'appuie sur la touche "retour". » Il nous est difficile de trancher, mais nous observons que les paragraphes réduits à une seule phrase sont très nombreux dans le corpus, même dans les lettres les plus réussies selon l'évaluation chiffrée de l'enseignante.

Nous pouvons alors émettre l'hypothèse que la ponctuation blanche remplace parfois le point: dans la lettre (III4Y, évaluée à 8), certains points finaux sont absents et les phrases sont en quelque sorte ponctuées par la présence de blancs. En outre, nous avons pu observer que dans les manuels à notre disposition, les exemples reprenaient 
ce modèle : une phrase, un paragraphe. Nous suspectons un apprentissage implicite par imprégnation.

41 Au final, nous ne pouvons que rester prudentes sur la fonction pragmatique de la ponctuation qui pourrait être illustrée par notre corpus. En revanche, de son côté, l'enseignante par un système de flèches, prouve à de nombreuses reprises l'attention particulière qu'elle porte à la mise en page de la lettre. Elle signale ainsi la nécessité de respecter des consignes typodispositionnelles qui visent le respect du modèle d'une lettre. Or, on peut s'interroger sur les savoirs réellement acquis par les élèves à ce sujet.

\section{Discussion}

42 Cette analyse descriptive de trente lettres de réclamation produites par des élèves de première gestion-administration, mise en parallèle avec les vingt-sept grilles d'évaluation remplies par l'enseignante, nous a permis de prouver qu'il existe bien des attendus vis-à-vis des élèves de LP en termes de savoir ponctuer. Ces attendus sont de deux ordres : d'abord, un respect de certaines règles syntaxiques de ponctuation qui semblent attirer l'attention de l'enseignante, ensuite, une compétence professionnelle qui consiste à respecter les normes du genre de la lettre, mais aussi à faire preuve d'une certaine efficacité communicative dans l'usage de signes de ponctuation ayant une fonction pragmatique. Notre analyse des réalisations des élèves, en réponse à cette tâche de rédaction proposée par leur enseignante, montre que ces derniers ont, pour la plupart, un certain nombre d'acquis du point de vue normatif. Ce résultat vient donc nuancer l'idée d'un rapport à la norme linguistique difficile chez les élèves de LP. En revanche, les réalisations des élèves dans le domaine pragmatique laissent à penser que, s'ils ont bien perçu des attentes formelles liées au genre du texte, certains élèves ont plus de mal à s'approcher d'un usage professionnel de la ponctuation, tourné vers le destinataire (Romain et coll., 2016).

Néanmoins, compte tenu du fait que notre analyse porte sur un texte produit en dehors d'une réelle situation de communication et n'inclue pas d'entretiens d'explicitation nous donnant accès au raisonnement des élèves face à la tâche, il nous est impossible de tirer des conclusions quant à l'étendue réelle de leur compétence dans le domaine. Il serait également intéressant, pour mieux mesurer le degré de maitrise des normes syntaxiques de ponctuation de ces mêmes élèves, de comparer ces emplois en production aux résultats obtenus aux autres items du texte qui portaient sur une application des règles de ponctuation en dehors du contexte de production. Enfin, notre étude n'impliquant que deux classes et une enseignante, les observations faites ici sont peu généralisables. De plus, nous n'avons accès au point de vue de l'enseignante que par le biais indirect de ses évaluations, la lettre de réclamation et la ponctuation n'ayant pas fait l'objet d'une séquence d'enseignement observable ou d'un entretien d'explicitation autour de cette tâche d'évaluation avec l'enseignante. Nous ne disposons d'aucune donnée quant à ses représentations de la ponctuation en tant qu'objet d'enseignement. Le seul constat que nous pouvons émettre est que cette composante du savoir-faire rédactionnel n'a pas été évoquée dans la demande initiale d'action pédagogique formulée par l'équipe enseignante de ce LP. Elle n'a pas non plus été mentionnée lors de notre enquête initiale dans les réponses que font les enseignantes sur ce qui fait un «bon » écrit, ce qu'elles évaluent en priorité ou ce qui met les élèves en difficulté. C'est d'ailleurs ce constat qui nous a amenées à émettre l'hypothèse qu'il y 
avait peut-être là une forme d'impensé didactique à interroger. Le manque de données concernant les représentations de l'enseignante et ses processus d'évaluation est sans aucun doute une des limites majeures de notre étude. Nous espérons cependant que cette attention particulière portée à la mise en œuvre d'un savoir ponctuer dans des textes d'élèves de LP peut ouvrir vers des perspectives didactiques (que nous précisons ci-après) concernant la place de la ponctuation dans l'enseignement de l'écrit au LP.

\section{Perspectives didactiques en guise de conclusion}

\subsection{Vers une approche différenciée des aspects syntaxiques}

Cette étude laisse entrevoir des savoir-faire, inégalement partagés dans la classe, qui constituent des «compétences ignorées» (Penloup, 2007), dans la mesure où elles ne font pas l'objet d'une attention particulière, de la part de l'enseignante comme de celle des élèves.

Or, elles pourraient servir, selon nous, de point d'appui à un travail de révision entre pairs (fondé sur les acquis des élèves). Cette approche collaborative des aspects normatifs du savoir ponctuer pourrait en effet, à condition de s'inscrire dans le cadre d'une pédagogie fortement différenciée, répondre aux besoins hétérogènes des élèves en matière de maitrise des normes syntaxiques de la ponctuation. Un tel travail, axé sur la nécessité de réviser ses textes, aurait également l'avantage de replacer les enjeux communicationnels au cœur de la tâche de production écrite.

\subsection{Vers un enseignement explicite du savoir ponctuer}

La manière dont la majorité des élèves organisent leur texte en paragraphes-phrases, sans que l'on puisse toutefois déterminer s'ils se conforment par imprégnation à une norme typodispositionnelle plus ou moins intériorisée ou s'ils construisent de réelles unités textuelles, nous amène également à envisager un enseignement plus visible et explicite des attendus en termes de ponctuation.

$\mathrm{Au}$ LP comme ailleurs, la ponctuation fait plus souvent l'objet d'un apprentissage par imprégnation que d'un enseignement explicite (Dufour, 2017). Or, on sait que l'enseignement implicite pose davantage de difficultés aux élèves moins bien pourvus en capital scolaire (Bautier \& Rayou, 2014) et peut constituer une source d'inégalités scolaires auprès de ce public particulier du LP. Si la ponctuation a déjà fait l'objet de plusieurs recherches en didactique de l'écrit (davantage centrés sur l'enseignement primaire que secondaire), en revanche, peu de travaux didactiques ont été, à ce jour, consacrés à la question du découpage en paragraphes bien que la linguistique ait permis des avancées dans ce champ qui devraient permettre une meilleure prise en compte des multiples composantes de la compétence scripturale dans l'enseignement (Adam \& Revaz, 1989; Arabyan, 2014). Les enseignants ne sont donc pas véritablement guidés dans la mise en place d'un enseignement explicite de la mise en paragraphes. Cela explique sans doute en grande partie le fait que, bien que la structure de la lettre soit évaluée par l'enseignante de notre étude, la mise en paragraphes ne constitue pas un item d'évaluation à part entière dans sa grille.

Il nous semble donc important que les enseignantes et les enseignants tout comme les élèves puissent considérer la ponctuation dans son ensemble (en tant que système) et 
dans la pluralité de ses fonctions comme un objet didactique à part entière qui trouverait sa place dans l'enseignement comme dans l'évaluation. La ponctuation pourrait, dans la dimension «méta-scripturale » qu'elle suppose (notamment en ce qui concerne la ponctuation blanche), constituer une entrée pour un travail réflexif mené en classe par les élèves sur leurs productions.

\subsection{La lettre de réclamation comme entrée dans l'enseignement de la ponctuation en LP?}

La fonction pragmatique de la ponctuation dans les lettres de réclamation apparait justement comme une bonne entrée pour cet enseignement. Considérer la manière dont la ponctuation va pouvoir servir la visée communicative de la lettre de réclamation serait parfaitement en accord avec les objectifs de formation de la filière.

Aborder la ponctuation par cette entrée pragmatique serait également une parfaite occasion de s'appuyer sur le rapport à l'écrit de ces élèves de LP qui conçoivent majoritairement une fonction communicative de l'écrit (De Amaral, à paraitre) pour les amener à s'intéresser à la construction du texte et d'autres éléments plus problématiques. Il s'agirait donc d'une première entrée pour les amener à réviser leurs textes.

\section{BIBLIOGRAPHIE}

ADAM, Jean-Michel \& REVAZ Françoise. (1989). Aspects de la structuration du texte descriptif : les marqueurs d'énumération et de reformulation. Langue française, 81, 59-98.

AdDIs, Dominique, Gauthier, Valérie, Koleosho Raji, Ayodele, Mullet, Sandrine \& RaVAine, Nathalie. (2016). Gestion Administration $1^{\text {re }}$ Bac pro. Delagrave.

ANIS, Jacques. (1983). Visibilité du texte poétique. Langue française, 59, 88-102.

ARABYAN, Marc (2014). La notion de paragraphe en rédaction : narration vs dissertation. Le français aujourd'hui, 187, 19-29. <https://doi.org/10.3917/lfa.187.0019>.

ARSENEAU, Rosianne. (2020). Comment la ponctuation se développe-t-elle chez les élèves ? Une revue systématique des recherches empiriques en classe de français langue d'enseignement. Bellaterra Journal of Teaching \& Learning Language \& Literature, 13, 1-20.

BARRÉ-DE MINIAC, Christine. (2001). L'écriture socio-professionnelle : un genre utile pour la didactique de l'écriture. Lidil, 23, 187-208.

BARRÉ-DE MINIAC, Christine. (2008). Le rapport à l'écriture : une notion à valeur heuristique. Dans S. Chartrand \& C. Blaser (dir.), Le rapport à l'écrit : un outil pour enseigner de l'école à l'université (p. 11-23). Presses universitaires de Namur.

BAUTIER, Élisabeth \& RAYOU, Patrick. (2014). Les inégalités d'apprentissage : programmes, pratiques et malentendus scolaires. Presses Universitaires de France. 
BESSONNAT, Daniel. (1991). Enseigner la... « ponctuation »? (!). Pratiques : linguistique, littérature, didactique, 70, 9-45.

CHARLOT, Bernard. (1999). Le rapport au savoir en milieu populaire : une recherche dans les lycées professionnels de banlieue. Anthropos.

CNESCO \& CIEP. (2016). De vraies solutions pour l'enseignement professionnel. Dossier de synthèse. $<$ www.cnesco.fr/fr/dossier-enseignement-professionnel/>.

DALHET, Véronique. (2003). Ponctuation et énonciation. Ibis Rouge Édition.

DABÈnE, Michel. (1991). Un modèle didactique de la compétence scripturale. Repères, 4, 9-22.

DE AMARAL, Cindy. (À paraitre). Représentations de l'écrit chez des élèves et enseignants en lycée professionnel : une mise en perspective contrastée. Étude de cas conduite sur deux classes des sections gestion-administration. SFERE.

DE AMARAL, Cindy. (2019). Écrire au lycée professionnel : obstacles et dispositifs de réapprentissage [Thèse de doctorat, nouveau régime]. Université Grenoble Alpes, Grenoble.

Dufour, Marie-Pierre. (2017). Éléments de réflexion sur l'enseignement de la ponctuation. Correspondance, 22,5 .

ELUERD, Roland. (2017). La ponctuation française : règles, usages et plaisir du texte. Éditions Garnier.

FAyol, Michel. (1997). Des idées au texte. Presses universitaires de France.

GUERNIER, Marie-Cécile. (2008). Discours d'enseignants sur les difficultés avec l'écrit des élèves de lycée professionnel. TransFormations, 1, 196-205.

Guernier, Marie-Cécile, BARRÉ-De Miniac, Christine, Mout, Tiphaine \& BrisSAud, Catherine. (2017). Ces lycéens en difficulté avec l'écrit et avec l'école. UGA Éditions.

JACQUES, Marie-Paule \& DE AMARAL, Cindy. (2018). Explorer les compétences rédactionnelles au lycée professionnel. SHS Web of Conferences, 46, 07008. <https://doi.org/10.1051/shsconf/ 20184607008>

JAGUENEAU, Liliane. (2000). La ponctuation dans un corpus épistolaire ordinaire. Dans J. Dürrenmatt (dir.), La ponctuation (p. 189-201). La Licorne.

JOYET, Angeline \& PLISSONNEAU, Gersende. (2017). Enseigner la grammaire en baccalauréat professionnel : défi ou défiance ? Le français aujourd'hui, 199, 51-61.

LAFONT-TERRANOVA, Jacqueline. (2009). Se construire, à l'école, comme sujet-écrivant : l'apport des ateliers d'écriture. Presses universitaires de Namur.

LAHIRE, Bernard. (2008). La raison scolaire. École et pratiques d'écriture, entre savoir et pouvoir. Presses universitaires de Rennes.

MAYNARD, Catherine, BRISSAUD, Catherine \& ARMAND, Françoise. (2018). Mise à l'essai d'un dispositif renouvelé d'enseignement de l'orthographe auprès d'élèves d'un lycée professionnel en France. SHS Web of Conferences, 46, 07002. <https://doi.org/10.1051/shsconf/20184607002>.

Paolacci, Véronique, BAin, Daniel \& Dufour, Marie-Pierre. (2016). L'enseignement de la ponctuation. Le cas de la virgule. Dans S. Chartrand (dir.), Mieux enseigner la grammaire. Pistes didactiques et activités (p. 225-348). ERPI.

PaOlacci, Véronique \& Rossi-Gensane, Nathalie. (2016). La question de la progressivité des apprentissages en production écrite à l'école élémentaire française : le cas de la segmentation en phrases. SHS Web of Conferences, 27, 07011. <https://doi.org/10.1051/shsconf/20162707011>. 
PAOLACCI, Véronique \& ROSSI-GENSANE, Nathalie. (2019). Les emplois de la virgule dans des écrits d'étudiants se destinant à devenir enseignants. Dans M. Niwese, J. Lafont-Terranova \& M. Jaubert (dir.), Écrire et faire écrire dans l'enseignement postobligatoire : enjeux, modèles et pratiques innovantes (p. 213-229). Presses universitaires du Septentrion.

PENLOUP, Marie-Claude. (2007). Les connaissances ignorées : approche pluridisciplinaire de ce que savent les élèves. Institut national de recherche pédagogique.

RomAin, Christina, ReY, Véronique \& PEREIRA, Marie-Emmanuelle. (2016). La fonction pragmatique de la ponctuation dans la rédaction professionnelle : une étude de cas. SHS Web of Conferences, 27, 02015. <https://doi.org/10.1051/shsconf/20162702015>.

TOURNIER, Claude. (1980). « Histoire des idées sur la ponctuation, des débuts de l'imprimerie à nos jours ». Langue française, 45, 28-40.

VÉDÉNINA, Ludmila G. (1980). La triple fonction de la ponctuation dans la phrase : syntaxique, communicative et sémantique. Langue française, 45, 60-76.

\section{ANNEXES}

Annexe 1. - Capture d'écran de la consigne du test telle qu'elle se présente à l'élève.

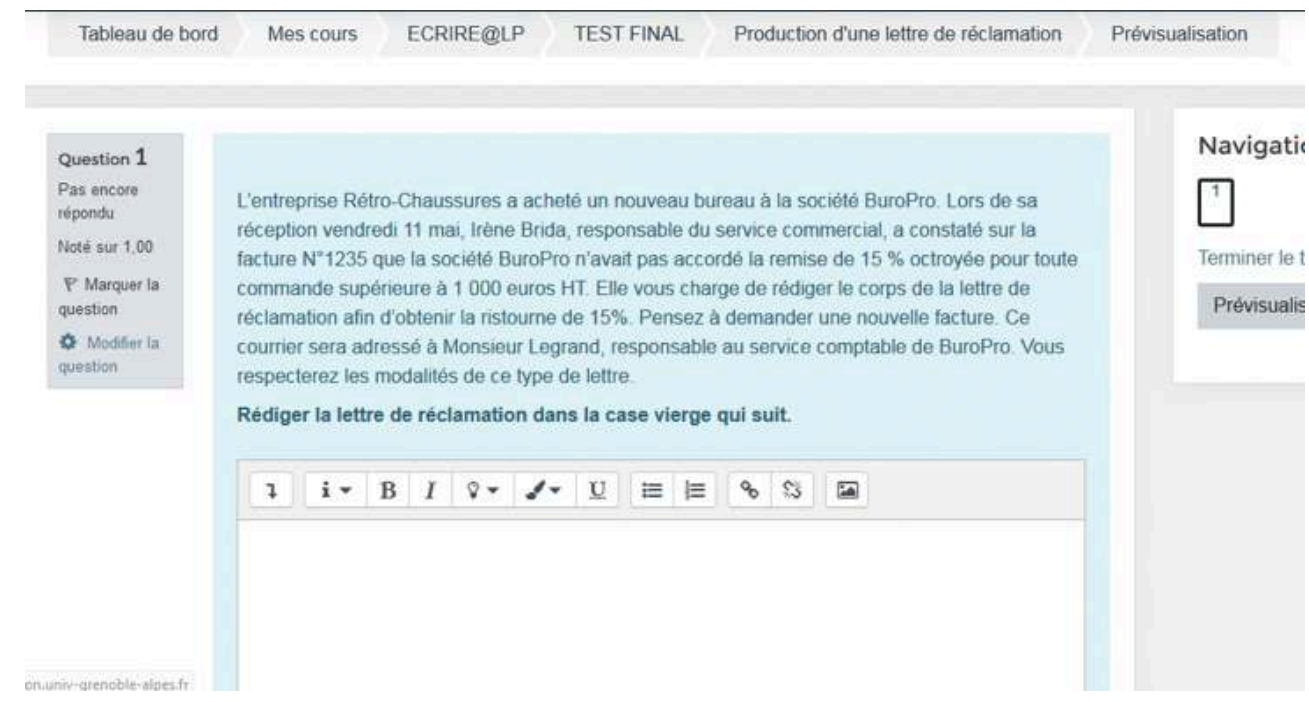


Annexe 2. - Exemple d'une grille d'évaluation de l'enseignante.

\begin{tabular}{|c|c|c|c|c|}
\hline & Degré 3 & Degré 2 & Degré 1 & \\
\hline Critères & 3 points & 2 points & 1-0 point & $\begin{array}{l}\text { NOMBRE DE } \\
\text { POINTS }\end{array}$ \\
\hline \multirow[t]{2}{*}{$\begin{array}{l}\text { Normes de } \\
\text { présentation }\end{array}$} & $\begin{array}{l}\text { - Les normes ont } \\
\text { été respectées en } \\
\text { grande partie }\end{array}$ & $\begin{array}{l}\text { - Les normes } \\
\text { n'ont été } \\
\text { respectées que } \\
\text { partiellement }\end{array}$ & $\begin{array}{l}\text { - Les normes } \\
\text { n'ont pas été } \\
\text { respectées }\end{array}$ & \\
\hline & $14-10$ points & 9.6 points & 5.0 points & \\
\hline \multirow[t]{2}{*}{ Contenu } & $\begin{array}{l}\text { - Les éléments } \\
\text { essentiels y } \\
\text { figurent }\end{array}$ & $\begin{array}{l}\text { - Les eléments } \\
\text { essentielsy } \\
\text { figurent } \\
\text { partiellement }\end{array}$ & $\begin{array}{l}\text { - Trop peu } \\
\text { d'éléments pour } \\
\text { qu'il y ait } \\
\text { construction de } \\
\text { sens }\end{array}$ & \\
\hline & 3 points & 2 points & $1-0$ point & \\
\hline $\begin{array}{l}\text { Construction / } \\
\text { Expression et } \\
\text { correction de la } \\
\text { langue française }\end{array}$ & $\begin{array}{l}\text { - L'ensemble } \\
\text { forme un texte en } \\
\text { grande partie } \\
\text { structuré } \\
\text { (paragraphe) et } \\
\text { cohérent, } \\
\text { enchaînement } \\
\text { assez logique des } \\
\text { eléments } \\
\text { essentiels } \\
\text { - Quelques } \\
\text { erreurs de } \\
\text { grammaire, } \\
\text { - Quelques } \\
\text { erreurs (syntaxe } \\
\text { et orthographe) }\end{array}$ & $\begin{array}{l}\text { - Des erreurs de } \\
\text { grammaire } \\
\text { - De nombreuses } \\
\text { erreurs (syntaxe } \\
\text { et orthographe) }\end{array}$ & $\begin{array}{l}\text { - Grammaire et } \\
\text { vocabulaire } \\
\text { insuffisants ou } \\
\text { inappropries } \\
\text { - Graves erreurs }\end{array}$ & I \\
\hline & & & Note sur 20 & \\
\hline
\end{tabular}

Annexe 3. - Capture d'écran d'une copie annotée, III9X, évaluée à 6.

Bonjour Monsieur LEGRAND,

¿e vous contacte afin de vous signalé que vous n'avez pas fait la remise de $15 \%$ sur la facture n`1235

$\mathrm{Al}^{\Omega}$ (orque notre commande avait atteint les $1000 \mathrm{\epsilon}$

Veuillez agrée nos salutation les plus sincère.

\section{NOTES}

1. Le système scolaire français se divise, à partir du lycée (pour les élèves âgés de 15 à 18 ans), en trois filières: générale, technologique et professionnelle. La filière générale offre un accès privilégié aux études supérieures tandis que les deux autres filières sont pensées comme plus professionnalisantes. Les filières technologiques étant plus sélectives, ce sont souvent les élèves les plus en difficulté à l'école et à l'écrit qui sont finalement orientés vers le lycée professionnel pour y préparer soit un CAP soit un baccalauréat professionnel (le baccalauréat professionnel permettant d'envisager une éventuelle poursuite d'étude dans des sections professionnalisantes de l'enseignement supérieur à hauteur de bac $+2 \mathrm{ou}+3$ le plus souvent).

2. Au sens où il ne s'agit pas seulement de tenir un discours sur la langue, mais aussi sur le texte et les processus d'écriture.

3. Pour une revue des travaux, voir Arseneau (2020).

4. Il suffit de lire ces deux phrases souvent citées dans les manuels scolaires : « Venez manger, les enfants! »vs « Venez manger les enfants! ».

5. «La ponctuation blanche ne se situera pas uniquement dans la séparation des mots, elle prendra complètement sens dans l'espace entier du support du texte. Il faut considérer les rôles 
particuliers de la mise en colonnes, des marges, des paragraphes, des alinéas, des lignes laissées blanches, etc. » (Eluerd, 2017, p. 135)

6. Les trois autres, considérées comme hors sujet, n'ont fait l'objet d'aucune annotation de l'enseignante (hormis l'attribution d'un zéro).

7. La séquence didactique au terme de laquelle se situe cette consigne dans le manuel intègre dans une simulation de "situation professionnelle" des savoirs rédactionnels qui lui sont subordonnés dans le référentiel de formation: rédiger une lettre de réclamation. L'approche didactique proposée dans les activités précédant cette tâche de rédaction consiste à faire surgir un «modèle " de la lettre de réclamation-type à partir d'une lecture analytique d'un texte modèle et d'éléments caractéristiques synthétisés dans un encadré "mémo " qui résume les attendus du genre « lettre de réclamation ». Ces pages correspondent à une entrée par les genres préconisée par le référentiel de formation et appliquée systématiquement par l'enseignante.

8. Cette séance, qui n'a pas été menée en classe auprès de ces élèves, mais qui participe des pratiques habituelles de l'enseignante de notre étude, nous semble constituer un indice important quant à la manière dont le genre « lettre de réclamation » est envisagé sur le plan didactique.

9. Nous utilisons un code d'anonymat pour désigner chaque élève (composé d'un chiffre romain indiquant la classe I ou III, un chiffre arabe et une lettre). Chaque légende indique l'identifiant de l'élève suivi de la note donnée par l'enseignante à la production.

10. C'est attesté par M. Grevisse et A. Goose, Le Bon usage, 1997, p. 162.

\section{RÉSUMÉS}

L'étude présentée consiste en une analyse seconde de données collectées lors d'un travail de thèse qui visait à améliorer la compétence rédactionnelle d'élèves de lycée professionnel scolarisés dans la filière gestion-administration (De Amaral, 2019). Il s'agit de réinterroger un corpus de textes initialement produits dans la phase de posttest de notre expérimentation dans une approche plus qualitative. Nous souhaitons en effet analyser avec un autre regard certains des textes élaborés en nous intéressant cette fois au savoir ponctuer que les élèves ont pu mettre en œuvre. Cette étude, qui part du postulat que la capacité de ces élèves à ponctuer est un élément essentiel pour le développement de leurs compétences rédactionnelles, cherche à valoriser les compétences mises en œuvre dans ce domaine particulier, mais aussi à identifier d'éventuelles difficultés. En mettant en parallèle notre analyse des textes d'élèves et l'évaluation proposée par l'enseignante au terme de ce projet de recherche, nous tenterons également de réfléchir à des pistes didactiques pour l'enseignement de la ponctuation au lycée professionnel.

The study proposes a second analysis of data collected during a doctoral research which aimed to improve the writing skills of technical college students enrolled in the management \& administration degree (De Amaral, 2019). We try to re-examine in a more qualitative approach a corpus of texts initially produced in the post-test phase of our experimentation. We would like to focus this time on the punctual skills of the students. This study, which is based on the premise that the ability of learners to punctuate is an essential element in the development of their writing skills, seeks to enhance the skills implemented in this particular area, but also to identify possible difficulties. By comparing our analysis of the students' texts with the assessment 
proposed by their teacher at the end of this research project, we will also try to give some didactic approaches for teaching punctuation at the technical College.

INDEX

Mots-clés : écriture, rapport à l'écrit, lycée professionnel, ponctuation et sa fonction pragmatique

Keywords : writing skills, relation to writing, technical college, punctuation pragmatics

\section{AUTEURS}

\section{CINDY DE AMARAL}

INSPÉ d'Évry, LIDILEM (Université Grenoble Alpes) et EMA (Gennevilliers)

deamaralcindy@gmail.com

\section{VÉRONIQUE PAOLACCI}

INSPÉ de Toulouse Pyrénées Occitanie, CLLE-ERSS (UMR 5260), Université Toulouse 2 Jean Jaurès

veronique.paolacci@univ-tlse2.fr 